\title{
Biocontrol potential of Pochonia chlamydosporia var. chlamydosporia isolates against Meloidogyne javanica on pistachio
}

\author{
M. Ebadi ${ }^{1}$, S. Fatemy ${ }^{2 *}$ and H. Riahi ${ }^{3}$
}

\begin{abstract}
The biocontrol potential of four Pochonia chlamydosporia var. chlamydosporia isolates on the root-knot nematode, Meloidogyne javanica was investigated on pistachio plants in a greenhouse experiment. Isolates were applied at 10,000, 5000 and 1000 chlamydospores per gram of soil; the latter two densities were being used for strain Pcc60 only and designated as PcCB and PccA, respectively. Plants were inoculated with a suspension containing 3000 eggs of M. javanica. Nematode reproduction was reduced to 57\% by Pcc20 and 36\% by Pcc10 and Pcc60C after 4 months. Pcc20 was the most promising isolate as the final egg population of nematodes was reduced by nearly 61 and $36 \%$ by Pcc10 and Pcc60C, respectively. All strains infected eggs on the roots to varying degrees. Pcc20 was the most effective colonizer of all the isolates, infecting 37\% of the eggs, while Pcc10 and Pcc60C as the second best colonizers caused an average infection rate of $17.5 \%$. The potential of the fungus was reduced at lower densities of spore inoculum. Semi-selective medium was used to re-isolate the fungi from soil and rhizosphere but was not able to detect Pcc60A at $1000 \mathrm{cfu} / \mathrm{g}$ soil, despite the fact that 3\% of the eggs were parasitized. The efficiency of the different isolates in controlling nematode reproduction parameters and their relationships in terms of survival and abundance is discussed further.
\end{abstract}

Keywords: Biological control, Pistacia vera, Root-knot nematode, Meloidogyne javanica, Survival, Pochonia chlamydosporia var. chlamydosporia

\section{Background}

Pistachio, Pistacia vera (Anacardiaceae) trees originated in the dry lands and desert climates of Asia Minor and are known in Iran as "Green Gold", being an important nutritional and economic commodity. Iran is the biggest producer of pistachio in the world, with approximately 346,000 ha of land under its cultivation and producing approximately 315,000 tons of yield annually (FAO 2016).

Plant parasitic nematodes are major constraints to agricultural production worldwide (Stirling 2014). In a list of the top ten rated plant parasitic nematodes, rootknot nematodes occupy the first position (Jones et al. 2013). Ten of the 98 described Meloidogyne species are agricultural pests whereas 5 species of cyst and 6 species

\footnotetext{
* Correspondence: sfatemy@yahoo.com

${ }^{2}$ Nematology Department, Iranian Research Institute of Plant Protection, Agricultural Research Education and Extension Organization, AREEO, No 1 , Yaman Ave, Tehran, Iran

Full list of author information is available at the end of the article
}

of lesion nematodes are of economic importance. Rootknot nematodes are obligate biotrophs of a wide range of plant species, the most important species being the tropical Meloidogyne arenaria, M. incognita and $M$. javanica and the temperate M. hapla (Jones et al. 2013). In Iran, M. incognita and M. javanica are the major economic species found in pistachio orchards (Banihashemi and Kheiri 1995). Their damage impact and its management is one of the major challenges for the Iranian pistachio industry.

The management practices used in agriculture must be ecologically sound and promote a soil food web capable of keeping populations of plant-parasitic nematodes below the economic damage threshold (Stirling 2014). Managing biological diversity, productivity and vitality are the essence of a sustainable agriculture (Lewandowski et al. 1999). Thus, practices of pest management should protect those soil organisms that play an important part in soil health sustainability (Stirling 2014). 
Intensive use of nematicides has led to increased microbial degradation of organophosphate and carbamate nematicides in soil and outbreaks of damage caused by parasitic nematodes (Hugo et al. 2014). The urge to find alternatives to chemical nematicides and the phasing out of methyl bromide fumigant caused a vast uprising in research towards ecofriendly measures like biological control of parasitic nematodes (Stirling 2014).

The fungus, Pochonia chlamydosporia var. chlamydosporia (Goddard) Gams \& Zare (Clavicipitaceae), is a facultative parasite of root-knot and cyst nematodes (Anastasiades et al. 2008; Moosavi et al. 2010; Carneiro et al. 2011 and Evans et al. 2017). The filamentous fungus is a saprotrophic in soil (Siddiqui et al. 2009) and is able to colonize roots as an endophyte of diverse species (Bordallo et al. 2002). It has been reported that $P$. chlamydosporia colonizes roots endophytically, thereby promoting plant growth and eliciting plant defenses (Lopez-Llorca et al. 2002).

Isolates of $P$. chlamydosporia var. chlamydosporia differ in their pathogenicity to nematodes, their ability to grow in the rhizosphere, in production of chlamydospores and in their temperature optima for growth (Irving and Kerry 1986). In addition, the host plant has a significant effect on the growth of the fungus in the rhizosphere (Bourne et al. 1996).

One of the features of egg-parasitic fungi is their ability to penetrate the chitin layer in the eggs. Further proof of this has been obtained in recent studies when chitosan was found during nematode egg infection, an advantage for the adaptation of biocontrol fungi to their natural environment (Aranda-Martinez et al. 2016). This feature of chitosan production has been taken further by Aranda-Martineza et al. (2017), showing that ethylene can be produced from chitosan using nematophagous and entomopathogenic fungi.

Most studies of the biocontrol ability of $P$. chlamydosporia against parasitic nematodes have been on annual crops, however, and reports on perennial crops are few (Stirling 2014).

The main goal of this study was to characterize four isolates of $P$. chlamydosporia var. chlamydosporia for future selection as a biocontrol agent against $M$. javanica on pistachio. In greenhouse conditions, whether these isolates are able to colonize roots, the following criteria were evaluated:

1) Their competence in colonizing egg masses on the roots; the degree to which they were able to reduce reproduction of M. javanica;

2) Their capability for establishment, survival and multiplication whether they could promote growth of pistachio plants.

\section{Methods}

Nematode inoculum

Nematodes used for the experiment were reared on tomato seedlings of cv Early Urbana inoculated with 5000 second-stage juveniles (J2) as explained in Ebadi et al. (2009). At harvest, plant roots were washed, cut into pieces, placed in a jar of $0.5 \%$ commercial $\mathrm{NaOCl}$ and shaken for $4 \mathrm{~min}$. The suspension was washed with tap water through 75 - and $20-\mu \mathrm{m}$ sieves and their numbers were counted with a counting slide under a light microscope (Hussey and Barker 1973).

\section{Fungal production}

The four strains of $P$. chlamydosporia var. chlamydosporia (Pcc isolated with the accession numbers of Pcc10, Pcc20, Pcc30 and Pcc60) used in this study had been maintained on corn meal agar at $5{ }^{\circ} \mathrm{C}$ in the Nematology Department Collection, Iranian Research Institute of Plant Protection, Tehran.

The fungal inoculum preparations were made according to the procedure of De Leij et al. (1993). Conical flasks were filled with a mixture of sand + milled barley $(1: 1 \mathrm{v} / \mathrm{v})$ and $30 \mathrm{ml}$ distilled water was added for each $100 \mathrm{~g}$ of mixture and autoclaved at $121{ }^{\circ} \mathrm{C}$ for $20 \mathrm{~min}$ on two consecutive days. Flasks were inoculated with plugs of isolates, kept at $25{ }^{\circ} \mathrm{C}$ and occasionally shaken for even growth. After 3 weeks, $5 \mathrm{~g}$ of the sand/barley substrate, mixed well with $100 \mathrm{ml}$ distilled water, was transferred to a blender (Waring) and blended for $2 \mathrm{~min}$. The contents were washed onto a $45-\mu \mathrm{m}$ aperture sieve, and the chlamydospores were collected on a nested $10-\mu \mathrm{m}$ sieve (De Leij et al. 1993). The chlamydospores were counted by a hemocytometer.

\section{Greenhouse experiment}

A suspension of washed chlamydospores was mixed with $50 \mathrm{~g}$ sterilized sand, added to $1 \mathrm{~kg}$ natural soil collected from a pistachio orchard, and the resulting mixture was used to fill a 14-cm diameter plastic pot, to give a final count of $1 \times 10^{4}$ chlamydospores/g soil. This spore density was used for all four fungal isolates and two further sets of chlamydospore densities were prepared to give $1 \times 10^{3}$ and $5 \times 10^{3}$ chlamydospores/g soil for isolate Pcc60 only and designated Pcc60A and Pcc60B respectively and Pcc60C $\left(1 \times 10^{4}\right.$ chlamydospores/g soil).

The nematode genera present in soil were mostly nonparasitic nematodes with very few Tylenchidae, and there were no root-knot nematodes present.

Pistachio cv Kaleghochi was chosen for the experiment. Prior to planting, seeds were disinfested for $4 \mathrm{~min}$ in $1 \% \mathrm{NaOCl}$, rinsed with sterile distilled water, immersed in $1 \%$ pentachloronitrobenzen, followed by soaking overnight in sterilized water and then pre- 
germinated in the dark on moist filter paper in Petri dishes.

One seedling was planted in each pot and allowed to establish for a month, when each relevant pot was inoculated with a suspension of nearly 3000 eggs of $M$. javanica added to three holes around each plant. Treatments included: nematode + isolate of Pcc10, Pcc20, Pcc30 and Pcc60C (10,000 cfu/g soil); nematode + isolate Pcc60B (5000 cfu/g soil), nematode + isolate Pcc60A $(1000 \mathrm{cfu} / \mathrm{g}$ soil), nematodes alone and pistachio alone. Each treatment was replicated five times, and the pots were arranged in a completely randomized design on a glasshouse bench, with average temperature of $27.5{ }^{\circ} \mathrm{C}$, and irrigated as required.

Plants were harvested after 4 months. Roots were washed in water, blotted gently dry and the fresh weights of the pistachio shoots and roots were taken. The numbers of galls or egg masses on roots were rated based on the $0-5$ scale of Hartman and Sasser (1985).

To determine nematode multiplication rates, eggs were extracted from the roots by the $\mathrm{NaOCl}$, using the same procedure as described above (Hussey and Barker 1973), treated roots were further processed in a blender to extract any possibly hidden eggs within the roots. The extracts were filtered through 75 - and $20-\mu \mathrm{m}$ sieves, and the numbers of eggs were estimated from the contents of the latter.

The populations of $\mathrm{J} 2$ were measured in $200 \mathrm{~g}$ soil from each pot of each treatment combination by means of modified Whitehead trays (Whitehead and Hemming 1965). The final total population density of healthy nematodes was calculated by combining the total numbers of $\mathrm{J} 2$ and healthy eggs. For estimation of the numbers of healthy eggs, the total numbers of infected eggs were subtracted from the total numbers of eggs. A reproduction factor was estimated by dividing the final nematode population density by the initial population density (Pf/Pi) (Ebadi et al. 2009).

To verify the percentage of egg infection, 10 egg masses/replicate (there were few egg masses at the time) were incubated in $0.05 \% \mathrm{NaOCl}$ between a glass slide and coverslip and observed under $\times 400$ magnification. To re-isolate and confirm identification of these infected eggs, a $0.2-\mathrm{ml}$ sub-sample of a suspension made from these eggs was taken, spread over the surface of $0.8 \%$ water agar, containing $50 \mathrm{ppm}$ each of tetracycline, chloramphenicol and streptomycin, and the grown hyphae were sub-cultured on PDA for further examination.

Viability and abundance of the fungi in soil and on the roots at the end of the experiment were checked, using a SSM (semi-selective medium) (De Leij and Kerry 1991). A sub-sample of $1 \mathrm{~g}$ soil from each replicate was added to $9 \mathrm{ml}$ of $0.05 \%$ sterile water agar and used to prepare dilution series of $10^{-1}$ to $10^{-4}$. An aliquot of $0.2 \mathrm{ml}$ of each dilution was transferred onto a 9-cm Petri dish containing SSM, with three replicates per dilution. Dishes were kept in an incubator at $25{ }^{\circ} \mathrm{C}$ for 1 or 2 weeks and the final numbers of cfu were counted.

Semi-selective medium contained the following: 37. $5 \mathrm{mg}$ carbendazim, $37.5 \mathrm{mg}$ thiabendazole, $75 \mathrm{mg}$ rose bengal, $17.5 \mathrm{mg} \mathrm{NaCl}, 50 \mathrm{mg}$ each of streptomycin sulphate, chlortetracycline hydrochloride and chloramphenicol, $3 \mathrm{ml}$ Triton X-100, and $17 \mathrm{~g}$ corn meal agar (Difco) in a liter of distilled water.

Roots of each replicate were cut into small pieces and then a $1 \mathrm{~g}$ sub-sample was rinsed with sterile distilled water, crushed with a sterilized pestle and mortar and added to $9 \mathrm{ml}$ of $0.05 \%$ sterile water agar solution. As in the soil test, a dilution series was prepared and the suspensions were transferred onto the SSM. Colonies were counted after 1 week (Bourne et al. 1996).

\section{Statistical analysis}

All data were subjected to analysis of variance (ANOVA) , using SPSS version 16. Data were checked for homogeneity of variance before being pooled. Data for percent egg infection, the reproduction factor and number of healthy eggs and $\mathrm{J} 2$ /pot were transformed to $\arcsin (x)$, sqrt $(x+0.5)$ and $\log (x+1)$, respectively, before ANOVA. Means were separated using the least significant difference (LSD) test $(P \leq 0.05)$.

\section{Results and discussion}

All fungal isolates were able to survive to the end of the experiment (Fig. 1). However, none of the isolates proliferated in the soil during the time of the experiment as the final density of their spores declined to less than the initial density of inoculum. For survival potential, the

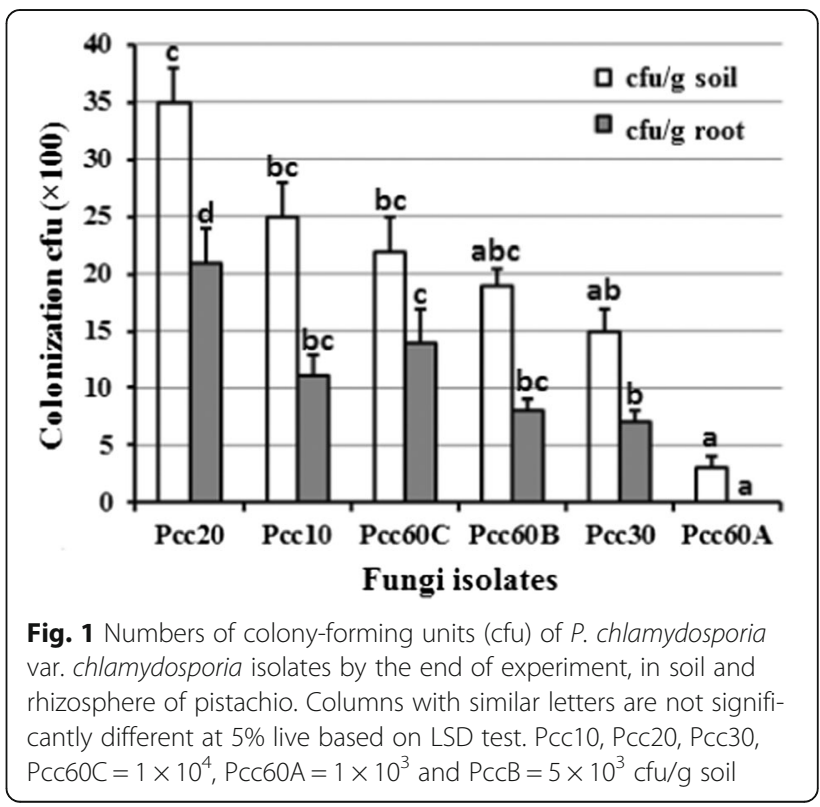


strains are ranked as Pcc20, Pcc10, Pcc60C and Pcc30 for having the highest to lowest final $\mathrm{cfu} / \mathrm{g}$ soil. The number of chlamydospores of Pochonia in pots, receiving $5000 \mathrm{cfu} / \mathrm{g}$ soil of Pcc60B as inoculum, was less than some isolates applied at $1 \times 10^{4} \mathrm{cfu} / \mathrm{g}$ soil, but was greater than the treatment with Pcc30. Strain Pcc60A survived during the experiment, although it had the least number of final spore/g soil of all strains.

Pochonia isolates colonized the root surface of pistachio and their numbers were greater for the strains of Pcc20 and Pcc60C (of average $1750 \mathrm{cfu}$ ) than other treatments (of average $650 \mathrm{cfu}$ ) (Fig. 1). Decreasing density of inoculum to $5 \times 10^{3}$ spores did not show any detrimental effect on survival of strain Pcc60B, which colonized roots and survived well till the end of the experiment. There was no colony growth on SSM from roots grown in soil treated with Pcc60A at the concentration of $1000 \mathrm{cfu} / \mathrm{g}$.

In the pot test, pistachio plants were infected with $M$. javanica but when untreated with fungus had the greatest number of galls (69.6) on their roots (Table 1). However, the numbers of galls decreased significantly $(P \leq 0$. $05)$ in the plants treated with fungal isolates, except for those of Pcc60A and Pcc60B, and were fewest (23.6) in the plants treated with Pcc20. All strains of Pcc-parasitized eggs within egg masses on roots but to varying degrees; the lightest rate of egg infection of 3 and 9\% was recorded in treatments of Pcc60A and Pcc60B respectively (Table 1). The rate of infection differed between the other treatments, although the only difference of significance was for Pcc20 which parasitized the greatest number of eggs on roots (37\%).

Nematode reproduction was significantly decreased in most fungus-treated plants, being below one in all pots, except for the Pcc60A and Pcc60B treatments (Table 1).

The final numbers of healthy eggs and $\mathrm{J} 2$ were reduced in most plants treated with fungal isolates, but not in the Pcc60A and Pcc60B treatments, which were not different than the untreated control $(P \leq 0.05)$ (Table 1).

Weights of pistachio-treated plants were not significantly different from the untreated plants (data not presented).

Based on our criteria, these fungal strains showed a potential as biocontrol agents for $M$. javanica on pistachio. The numbers of galls produced were fewest in Pcc20- and Pcc10-treated plants. The final egg population of nematodes was reduced by $61 \%$ by strain Pcc 20 and $36 \%$ by Pcc10 and Pcc60C. Nematode reproduction was reduced to 57 and $36 \%$ by Pcc 20 and Pcc10, respectively, and also it reached to $36 \%$ by Pcc60C. In previous work, these strains have colonized more than $80 \%$ of the $M$. javanica eggs on water agar (Ebadi et al. 2009 and Moosavi et al. 2010). They also decreased infection levels of $M$. javanica on tomato between 29 and 93\% (Moosavi et al. 2010). Strain of Pcc60 has lowered the $H$. schachtii population to $50 \%$ on sugar beet (Ayatollahy et al. 2008). Interesting results have also been obtained with these isolates of Pcc in recent pot trials on Globodera rostochiensis Woll.; isolates penetrated cysts and killed nearly $90 \%$ of the eggs in vitro (Dehghan Nasrabad and Fatemy 2016).

Tested strains, with some reservation, were able to control infections of the mentioned nematodes on different crops, despite the fact that they were initially isolated from sugar beet cyst nematode. Host preference in isolates from cyst and root-knot nematodes was reported by Mauchline et al. (2004) and Manzanilla-Lopez et al. (2011). The association has been related to specific amino acid polymorphisms in a fungal serine protease, VCP1, involved in the infection of nematode eggs (Morton et al. 2003). The enzyme degrades the proteinaceous vitelline membrane, the outer layer of the nematode eggshell, which is qualitatively different in root-knot and cyst nematodes (Morton et al. 2004). Under some circumstances, different isolates of Pcc and P. chlamydosporia var. catenulata failed to reduce gall

Table 1 The effect of Pochonia chlamydosporia var. chlamydosporia isolates on Meloidogyne javanica populations, survival of the fungus isolates and their colonization of roots of pistachio after 4 months in the greenhouse ( $n=5$ means \pm S.E)

\begin{tabular}{|c|c|c|c|c|c|c|c|}
\hline Treatments & Gall no./g root & Gall index & $\begin{array}{l}\text { Egg infection \% } \\
\text { (arc sin) }\end{array}$ & $\begin{array}{l}\mathrm{Pf} / \mathrm{Pi} \\
(\mathrm{sqrt} x+0.5)\end{array}$ & J2/pot & $\begin{array}{l}\text { Healthy egg and } J 2 / \text { pot } \\
(\log x+1)\end{array}$ & $\%$ control \\
\hline Nematode & $69.6 \pm 2.63 \mathrm{a}$ & 4 & $0 \mathrm{C}$ & $1.40(1.37) \pm 0.2 \mathrm{a}$ & $273 \pm 29$ & $4643(3.66) \pm 0.3 a$ & - \\
\hline Nematode + Pcc10 & $26.6 \pm 1.21 \mathrm{~d}$ & 3 & $19(0.1) \pm 0.02 b$ & $0.90(1.18) \pm 0.4 \mathrm{C}$ & $267 \pm 18$ & $2980(3.47) \pm 0.2 c$ & 36 \\
\hline Nematode + Pcc20 & $23.6 \pm 1.53 \mathrm{~d}$ & 3 & $37(0.4) \pm 0.01 \mathrm{a}$ & $0.55(1.02) \pm 02 \mathrm{~d}$ & $167 \pm 24$ & $1805(3.25) \pm 0.1 d$ & 61 \\
\hline Nematode + Pcc30 & $33.2 \pm 2.08 c$ & 4 & $16(0.1) \pm 0.01 \mathrm{~b}$ & $0.98(1.21) \pm 0.3 c$ & $333+32$ & $3253(3.51) \pm 0.2 c$ & 30 \\
\hline Nematode + Pcc60A & $64.0 \pm 1.84 \mathrm{a}$ & 4 & $3(0.02) \pm 0.04 c$ & $1.37(1.35) \pm 0.2 \mathrm{a}$ & $400 \pm 36$ & $4531(3.62) \pm 0.5 a$ & 2.5 \\
\hline Nematode + Pcc60B & $56.4 \pm 2.14 \mathrm{a}$ & 4 & $9(0.1) \pm 0.02 b$ & $1.20(1.28) \pm 0.3 b$ & $300 \pm 42$ & $3969(3.55) \pm 0.3 b$ & 15 \\
\hline Nematode + Pcc60C & $39.4 \pm 2.03 b$ & 4 & $16(0.2) \pm 0.03 b$ & $0.92(1.19) \pm 0.2 \mathrm{C}$ & $233 \pm 26$ & $3041(3.47) \pm 0.5 c$ & 35 \\
\hline \multirow[t]{2}{*}{ Pistachio } & - & - & - & - & - & - & - \\
\hline & $P<0.05($ SED $=3.01)$ & n.s. & $P<0.05(\mathrm{SED}=0.01)$ & $P<0.05($ SED $=0.06)$ & n.s. & $P<0.05(\mathrm{SED}=0.06)$ & \\
\hline
\end{tabular}

Data in parenthesis were transformed before being subjected to ANOVA; numbers followed by the same letter in the columns are not significantly different at $5 \%$ level according to LSD test; Pf/Pi $=$ ratio of final to initial population density, $\%$ control: control-treatment/control $\times 100$. Pcc10, Pcc20, Pcc30, Pcc60C $=1 \times 10^{4}$, $\mathrm{Pcc} 60 \mathrm{~A}=1 \times 10^{3}$ and $\mathrm{PccB}=5 \times 10^{3} \mathrm{cfu} / \mathrm{g}$ soil 
and egg mass indexes of M. enterolobii Yang and Eisenback on tomato or banana (Silva et al. 2017).

Selection of an efficient biocontrol agent is based on whether it kills nematodes or prevents them from causing damage to their hosts. Thus, the biocontrol agent must be re-isolated from soil or nematodes by appropriate and effective means to differentiate it from naturally occurring strains (Stirling 2014). After 4 months of the present experiment, fungal isolates survived in soil and established on the roots of pistachio. There were differences among strains; Pcc20 was the most efficient colonizer of roots, whereas Pcc10 and Pcc60C showed less potential in this respect. However, none of the isolates multiplied during the period of the experiment where their numbers had decreased at the end of the trial to be 65 and $85 \%$, respectively.

The relation between nematode egg infection and abundance of $P$. chlamydosporia in the rhizosphere is complicated (Atkins et al. 2009). Isolate Pcc60A (applied at $1 \times$ $10^{3} \mathrm{cfu} / \mathrm{g}$ soil) did not grow on SSM from treated roots, although it infected $3 \%$ of the eggs. The reason could be that its abundance on roots was not great enough to be detected by this procedure-perhaps more advanced DNAbased techniques could have done so. Estimates of fungal density based on solid media only may not be reliable since the differentiation between colonies grown from mycelium or spore was not possible; also, the estimation is further complicated for P. chlamydosporia, that produced both conidia and chlamydospores (Manzanilla-Lopez et al. 2009). Quantitative PCR (Mauchline et al. 2002) and quantitative real-time PCR (Atkins et al. 2005) have provided more accurate and complementary information on abundance of filamentous nematophagous fungi. However, densities less than 10,000 cfu inoculum (as in the case of Pcc60B and Pcc60A) were less efficient in controlling nematode density.

Compared to other strains, Pcc30 was a weak biocontrol agent and colonizer of soil and rhizosphere of pistachio. Differences observed in the tested isolates' pathogenicity and abundance on roots and in soil have also been experienced by others. Isolates of P. chlamydosporia differ in their ability to control Meloidogyne (Medina-Canales et al. 2014).

Some species of Pochonia can penetrate roots endophytically (Lopez-Llorca et al. 2002). This type of colonization by this fungus has been found to promote growth of the host plant (Monfort et al. 2005). Most of these assessments have been done on annual plants; in the obtained findings, the weight of pistachio cv Kaleghochi plants was insignificantly increased by any of the Pcc strains and weights of treated plants did not differ from untreated plants (data not shown). Since the endophytic penetration of these isolates was not measured and the literature on the subject on perennial crops is scarce, it would be interesting to further look at this aspect of the relationship in future studies.
Involvement of different enzymes (proteases, esterases, lipases and chitinases) in the infection process was observed (Morton et al. 2004). However, in spite of high infection levels of M. javanica eggs in vitro by Pcc, J2s were not paralyzed when exposed to culture filtrates of the tested isolates under laboratory conditions (Ebadi et al. 2009). Esteves et al. (2009) could not correlate enzyme activity with egg parasitism or rhizosphere colonization in vitro.

Experimental data on biocontrol activity of nematophagous fungi in orchards are minimal. In a kiwifruit orchard, populations of $P$. chlamydosporia and Purpureocillium lilacinum were dominant species infecting root-knot nematodes (Mertens and Stirling 1993). Chlamydospores are the survival stage of fungi and their germination is triggered by nutrients leaking from roots, or by the presence of organic matter (De Leij et al. 1993). P. chlamydosporia is a saprotrophic in soil and could grow on crop residues (Dallemole-Giaretta et al. 2011). The continual presence of endoparasitic nematodes in an undisturbed environment such as perennial crops provides a food source for $P$. chlamydosporia, while during the unfavorable season of winter, the fungus would survive by switching to the saprophytic stage using organic matter instead of nematode hosts (Stirling 2014).

\section{Conclusion}

The ability to survive, colonize roots and restrict nematode multiplication were encouraging in some of the isolates. The isolate of Pcc20 has the most pathogenic effects, while Pcc10 and Pcc60C were the moderate ones of Pcc and these isolates could be selected for further work in the natural environment.

\section{Acknowledgements}

This research was supported by the Iranian Research Institute of Plant Protection, Agricultural Research Education and Extension Organization, AREO, Tehran, Iran. The authors would like to thank Prof. K. Evans, formerly at Rothamsted Research, UK, for critically reviewing the manuscript.

\section{Funding}

Iranian Research Institute of Plant Protection has supported this research and all the financial means.

\section{Availability of data and materials}

Data will not be shared, because our institute does not allow it.

\section{Authors' contributions}

First author ME is responsible for implementing the experimental work. Second author SF is responsible for designing and supervising the study, revising the paper scientifically, and checking analysis and interpretation of data. Third author HR is responsible for the general co-operation and contribution to the study. All authors read and approved the final manuscript.

\section{Competing interests}

The authors declare that they have no competing interests.

\section{Author details}

${ }^{1}$ Faculty of Basic Sciences, Azarbaijan Shahid Madani University, Tabriz, Iran. ${ }^{2}$ Nematology Department, Iranian Research Institute of Plant Protection, Agricultural Research Education and Extension Organization, AREEO, No 1, 
Yaman Ave, Tehran, Iran. ${ }^{3}$ Technical and Engineering Campus of Shahid Beheshti University, Tehran, Iran.

\section{Received: 7 December 2017 Accepted: 23 April 2018 Published online: 15 May 2018}

\section{References}

Anastasiades IA, Giannakou IO, Prophetou-Athanasiadou DA, Gowen SR (2008) The combined effect of the application of a biocontrol agent Paecilomyces lilacinus, with various practices for the control of root-knot nematodes. Crop Prot 27:352-361

Aranda-Martinez A, Lenfant N, Escudero N, Zavala-Gonzalea EA, Henrissat B, Lopez-Llorca LV (2016) CAZyme content of Pochonia chlamydosporia reflects that chitin and chitosan modification are involved in nematode parasitism. Environ Microbiol 18:4200-4215

Aranda-Martineza A, Naranjo Ortizb MA, Abihssira Garcíaa IS, Zavala-Gonzaleza EA, Lopez-Llorca LV (2017) Ethanol production from chitosan by the nematophagous fungus Pochonia chlamydosporia and the entomopathogenic fungi Metarhizium anisopliae and Beauveria bassiana. Microbiol Res 204:30-39

Atkins SD, Clark I, Pande S, Hirsch PR, Kerry BR (2005) The use of real-time PCR and species-specific primers for the identification and monitoring of Paecilomyces lilacinus. FEMS Microbiol Ecol 51:257-264

Atkins SD, Peteira B, Clark IM, Kerry BR, Hirsch PR (2009) Use of real-time quantitative PCR to investigate root and gall colonisation by co-inoculated isolates of the nematophagous fungus Pochonia chlamydosporia. Ann Appl Biol 155:143-152

Ayatollahy E, Fatemy S, Etebarian H (2008) Potential for biological control of Heterodera schachtii by Pochonia chlamydosporia var. chlamydosporia on sugar beet. Biocontrol Sci Tech 18:157-167

Banihashemi Z, Kheiri A (1995) The occurrence of root knot nematode (Meloidogyne javanica) on pistachio in Damghan. Iranian J Plant Pathol 31:37-38

Bordallo JJ, Lopez-Llorca LV, Jansson HB, Salinas J, Persmark L, Asensio L (2002) Colonization of plant roots by egg-parasitic and nematode-trapping fungi. New Phytol 154:491-499

Bourne JM, Kerry BR, De Leij FAAM (1996) The importance of the host plant on the interaction between root-knot nematodes (Meloidogyne spp.) and the nematophagous fangus Verticillium chlamydosporium Goddard. Biocontrol Sci Tech 6:539-548

Carneiro RMDG, Hidalgo-Diaz L, Martins I, Silva KFAS, Souza MG, Tigano MS (2011) Effect of nematophagous fungi on reproduction of Meloidogyne enterolobii on guava (Psidium guajava L.) plants. Nematology 13:721-728

Dallemole-Giaretta R, de Freitas LG, Lopes EA, Ferraz S, de Podesta GS, Agnes EL (2011) Cover crops and Pochonia chlamydosporia for the control of Meloidogyne javanica. Nematology 13:919-926

De Leij FAAM, Kerry BR (1991) The nematophagous fungus Verticillium chlamydosporium as a potential biological control agent for Meloidogyne arenaria. Revue de Nematologie 14:159-164

De Leij FAAM, Kerry BR, Dennehy JA (1993) Verticillium chlamydosporium as a biological control agent for Meloidogyne incognita and M. hapla in pot and microplot tests. Nematologica 39:115-126

Dehghan Nasrabad A, Fatemy S (2016) Effect of Pochonia chlamydosporia var. chlamydosporia on potato cyst nematode, vol 89. 3rd National meeting on Biocontrol in Agriculture and Natural Resources, 2-3 Feb, Ferdowsi University, Mashad

Ebadi M, Fatemy S, Riahi H (2009) Evaluation of the efficacy of Pochonia chlamydosporia var. chlamydosporia as a biological control agent of Meloidogyn javanica on pistachio. Biocontrol Sci Tech 19:689-700

Esteves I, Peteira B, Atkins SD, Magan N, Kerry BR (2009) Production of extracellular enzymes by different isolates of Pochonia chlamydosporia. Mycol Res 113:867-876

Evans K, Manzanilla-Lopez RH, Lopez-Llorca LV (2017) Introduction (historical and overview). In: Manzanilla-López RH, Lopez-Llorca LV (eds) Perspectives in sustainable nematode management through Pochonia chlamydosporia applications for root and rhizosphere health. Sustainability in Plant and Crop Protection, Springer, pp 3-19

FAO (2016) FAOSTAT. Food and Agriculture Organization of the United Nations. Rome Hartman KM, Sasser JN (1985) Identification of Meloidogyne species on the basis of differential host test and perineal pattern morphology. In: K.R. Baker, C.C. Carter, J.N. Sasser, (eds.), An advanced treatise on Meloidogyne. Vol. 2, Methodology, North Carolina State University: pp. 69-77
Hugo HJ, Mouton C, Malan AP (2014) Accelerated microbial degradation of nematicides in vineyard and orchard soils. S Afr J Enol Viticulture 35:157-167

Hussey RS, Barker KR (1973) A comparison of methods of collecting inocula of Meloidogyne spp., including a new technique. Plant Disease Reporter 57 . 1025-1028

Irving F, Kerry BR (1986) Variation between strains of the nematophagous fungus, Verticillium chlamydosporium Goddard. II. Factors affecting parasitism of cyst nematode eggs. Nematologica 32:474-485

Jones JT, Haegeman A, Danchin EGJ, Gaur HS, Helder J, Jones MGK, Kikuchi T, Manzanilla-Lopez R, Palomares-Rius JE, Wesemael WML, Perry RN (2013) Top 10 plant parasitic nematodes in molecular plant pathology. Mol Plant Pathol 14:946-961

Lewandowski I, Hardtlein M, Kaltschmitt M (1999) Sustainable crop production: definition and methodological approach for assessing and implementing sustainability. Crop Sci 39:184-193

Lopez-Llorca LV, Bordallo JJ, Monfort E, Lopez-Serna ML (2002) Use of light and scanning electron microscopy to examine colonisation of barley rhizosphere by the nematophagous fungus Verticillium chlamydosporium. Micron 3:61-67

Manzanilla-Lopez RH, Atkins SD, Clark IM, Kerry BR, Hirsch PR (2009) Measuring abundance, diversity and parasitic ability in two populations of the nematophagous fungus Pochonia chlamydosporia var. chlamydosporia. Biocontrol Sci Tech 19:391-406

Manzanilla-Lopez RH, Esteves I, Powers SJ, Kerry BR (2011) Effects of crop plants on abundance of Pochonia chlamydosporia and other fungal parasites of root-knot and potato cyst nematodes. Ann Appl Biol 159:118-129

Mauchline TH, Kerry BR, Hirsch PR (2002) Quantification in soil and the rhizosphere of the nematophagous fungus Verticillium chlamydosporium by competitive PCR and comparison with selective plating. Appl Environ Microbiol 68:1846-1853

Mauchline TH, Kerry BR, Hirsch PR (2004) The biocontrol fungus Pochonia chlamydosporia shows nematode host preference at the infraspecific level. Mycol Res 108:161-169

Medina-Canales MG, Rodriguez-Tovar AV, Manzanilla-Lopez RH, Zúñiga G, TovarSoto A (2014) Identification and molecular characterisation of new Mexican isolates of Pochonia chlamydosporia for the management of Meloidogyne spp. Biocontrol Sci Tech 24:1-21

Mertens MCA, Stirling GR (1993) Parasitism of Meloidogyne spp. on grape and kiwifruit by the fungal egg parasites Paecilomyces lilacinus and Verticillium chlamydosporium. Nematologica 39:400-410

Monfort E, Lopez-Llorca LV, Jansson HB, Salinas J, Park JO, Sivasithamparam K (2005) Colonisation of seminal roots of wheat and barley by egg-parasitic nematophagous fungi and their effects on Gaemannomyces graminis var. tritici and development of root-rot. Soil Biol Biochem 37:1229-1235

Moosavi M, Zare R, Zamanizadeh H, Fatemy S (2010) Pathogenicity of Pochonia species on eggs of Meloidogyne javanica. J Invertebr Pathol 104:125-133

Morton CO, Hirsch PR, Kerry BR (2004) Infection of plant-parasitic nematodes by nematophagous fungi - a review of the application of molecular biology to understand infection processes and to improve biological control. Nematology 6:161-170

Morton CO, Hirsch PR, Peberdy JP, Kerry BR (2003) Cloning and genetic variation in protease VCP1 from the Nematophagous fungus Pochonia chlamydosporia. Mycol Res 107:38-46

Siddiqui IA, Siddiqui SD, Atkins BR, Kerry BR (2009) Relationship between saprotrophic growth in soil of different biotypes of Pochonia chlamydosporia and the infection of nematode eggs. Annals of Applied Biology 155:131-141

Silva SD, Carneiro RMDG, Faria M, Souza DA, Monnerat RG, Lopes RB (2017) Evaluation of Pochonia chlamydosporia and Purpureocillium lilacinum for suppression of Meloidogyne enterolobii on tomato and banana. J Nematol 49(1):77-85

Stirling GR (2014) Biological control as a component of integrated nematode management: the way forward. In: Stirling GR (ed) Biological control of PPN: soil ecosystem management in sustainable agriculture, second revised edition. CABI, UK, pp 393-407

Whitehead AG, Hemming JR (1965) A comparison of some quantitative methods of extracting small vermiform nematodes from soil. Ann Appl Biol 55:25-38 